\title{
Simplest cosmological model with the scalar field II. Influence of cosmological constant
}

\author{
A. Yu. Kamenshchik ${ }^{1 \dagger}$, I. M. Khalatnikov ${ }^{1,2 \dagger}$ and A. V. Toporensky ${ }^{3 *}$ \\ ${ }^{1}$ L. D. Landau Institute for Theoretical Physics, Russian Academy of Sciences, \\ Kosygin Street 2, Moscow, 117334, Russia \\ ${ }^{2}$ Tel Aviv University, Raymond and Sackler Faculty of Exact Sciences, School of \\ Physics and Astronomy, Ramat Aviv, 69978, Israel \\ ${ }^{3}$ Sternberg Astronomical Institute, Moscow University, Moscow, 119899, Russia
}

Continuing the investigation of the simplest cosmological model with the massive real scalar non-interacting inflaton field minimally coupled to gravity we study an influence of the cosmological constant on the behaviour of trajectories in closed minisuperspace Friedmann-Robertson-Walker model. The transition from chaotic to regular behaviour for large values of cosmological constant is discussed. Combining numerical calculations with qualitative analysis both in configuration and phase space we present a convenient classification of trajectories.

PACS: $98.80 . \mathrm{Hw}, 98.80 . \mathrm{Bp}$

$\dagger$ Electronic mail: kamen@landau.ac.ru

† Electronic mail: khalat@landau.ac.ru

* Electronic mail: lesha@sai.msu.su 


\section{Introduction}

This paper continues investigation of our previous paper ${ }^{1}$ where we studied the dynamics of the cosmological model, including gravity and minimally coupled scalar field with simple potential including only massive term. The dynamics of the minisuperspace cosmological models with the massive real scalar field for the flat, open and closed Friedmann universes was studied in papers ${ }^{2,3}$ in terms of phase space. It appeared that the dynamics of closed model is more complicated than that of open and flat models. This dynamics allows the transitions from expansion to contraction and the existence of points of maximal expansion and minimal contraction in contrast with cases of open and flat cosmologies. Moreover, closed spherically symmetric models cannot expand infinitely and should have the points of maximal expansion provided the matter in the model under consideration satisfies the condition of energodominance ${ }^{4}$. The presence of points of maximal expansion and minimal contraction opens the possibility for the existence of the trajectories escaping singularity and oscillating between turning points ${ }^{5}$. The possibility of existence of such trajectories or non-singular universes filled with scalar field was discussed also earlier in Ref. 6. Recently such a dynamics was studed from the viewpoint of theory of dynamical chaos ${ }^{12}$.

Our previous work ${ }^{1}$ was devoted to the classification of trajectories in closed model and to the study the idea about the fractal set of infinitely bouncing universes which was put forward in Ref. 5. Here we consider more rich model where cosmological constant is included into consideration. Dynamics in such a model is rather peculiar and is of interest from mathematical point of view. Besides, some attempts to reconstruct the potential of inflaton field studying the anysotropy of 
relic cosmic background radiation testify in favor of the existence of constant term in the power expansion of this potential ${ }^{7}$ :

$$
V(\varphi)=V_{0}+\frac{m^{2} \varphi^{2}}{2}+\cdots, \quad V_{0} \neq 0
$$

Apparently, $V_{0}$ plays role of cosmological constant $\Lambda$.

Thus, we have an action

$$
S=\int d^{4} x \sqrt{-g}\left\{\frac{m_{P}^{2}}{16 \pi}(R-2 \Lambda)+\frac{1}{2} g^{\mu \nu} \partial_{\mu} \varphi \partial_{\nu} \varphi-\frac{1}{2} m^{2} \varphi^{2}\right\},
$$

where $m_{P}$ is Planck mass. The presence of cosmological constant $\Lambda$ makes possible an existence of trajectories without points of maximal expansion, i.e. the trajectories which begin and end in DeSitter regime

$$
\begin{aligned}
& a(t) \sim \exp (-H t), \quad t \rightarrow-\infty ; \\
& a(t) \sim \exp (H t), \quad t \rightarrow \infty
\end{aligned}
$$

or the trajectories which begin in singularity and end in DeSitter expansion or vice versa. Here, in Eq. (1.2) $H$ denotes Hubble constant

$$
H=\sqrt{\frac{\Lambda}{3}}
$$

It is convenient to begin the consideration of possible classes of trajectories with an investigation of the simplest cosmological model when scalar field is massless, but cosmological constant $\Lambda \neq 0$ (the model with $m=0, \Lambda=0$ was studied in some detail in Refs. 8,1,9). The second section of our paper will be devoted to this case. The class of trajectories travelling between sigularities with possible multiple bounces will be described in third section in terms of configuration space. In fourth section we consider the trajectories of two types DeSitter-singularity and DeSitterDeSitter and the surface separating them in phase space. 


\section{Massless model with cosmological constant}

Equations of motion can be now written down in the following form

$$
\dot{y}=-3 y z
$$

and

$$
\dot{z}=z^{2}+\frac{\Lambda}{3}-\frac{8 \pi y^{2}}{3 m_{P}^{2}}
$$

where we have introduced notations

$$
y \equiv \dot{\varphi}, \quad z \equiv \frac{\dot{a}}{a}
$$

and dots denote differentiation in respect with time. First integral of motion looks as follows:

$$
z^{2}+\frac{1}{a^{2}}=\frac{4 \pi y^{2}}{3 m_{P}^{2}}+\frac{\Lambda}{3}
$$

As far as the variable $\varphi$ is not included into Eqs. (2.3)-(2.5) the corresponding dynamical system can be represented by the diagram of two-dimensional phase space with coordinates $(y, z)$, see Fig. 1. Here two hyperbola

$$
z= \pm \sqrt{\frac{4 \pi y^{2}}{3 m_{P}^{2}}+\frac{\Lambda}{3}}
$$

separate the part of phase space accessible for the trajectories of closed and open Friedmann - Robertson - Walker model (cf. Refs. 2,3). There are four special points: points $A$ and $C$ are attracting and repelling nodes respectively, while points $B$ and $D$ are saddle ones. The coordinates of these points have the following values:

$$
\begin{aligned}
& y_{A}=0, z_{A}=\sqrt{\frac{\Lambda}{3}} ; y_{B}=\sqrt{\frac{m_{P}^{2} \Lambda}{8 \pi}}, z_{B}=0 \\
& y_{C}=0, z_{C}=-\sqrt{\frac{\Lambda}{3}} ; y_{D}=-\sqrt{\frac{m_{P}^{2} \Lambda}{8 \pi}}, z_{D}=0 .
\end{aligned}
$$


The region $I$ bounded by trajectories connecting the points $A$ and $B, B$ and $C$, $C$ and $D, D$ and $A$ is covered by trajectories which begins at point $C$, i.e since contraction according to DeSitter exponential law at $t=-\infty$, then have a bounce or in other words go through the point of minimal contraction and end in the point A corresponding to DeSitter expansion.

The region $I I$ contains trajectories expanding from singularity until DeSitter regime while the region $I I I$ contains trajectories contracting from DeSitter point $C$ to singularity. Finally, the region $I V$ contains trajectories which begin expansion from singularity, then go through the points of maximal expansion and then return to singularity again. The regions $I I$ and $I I I$ are separated from the region $I V$ by trajectories connecting saddle points $B$ and $D$ with singularity.

It is interesting to notice that the picture analogous to that described above was obtained earlier for the hydrodynamical matter in the presence of cosmological constant in Ref. 10. The diagram similar to diagram in Fig.1 was constructed in the coordinates $\varepsilon$ (energy) and $z$. The stationary unstable solutions were obtained in Ref. 10 as well. Such a coincidence is quite natural because massless scalar field corresponds to the hydrodynamical matter with equation of state $\varepsilon=p$, where $p$ is pressure.

Inclusion of mass of scalar field makes the picture more complicated. Phase space becomes 3-dimensional and the opportunity of multiple bounces or passages via points of maximal expansion arises. Besides, the saddle points $B$ and $D$ disappear. Nevertheless, one can speak roughly about three regimes again: they can be called "singularity-singularity", "singularity-DeSitter" and "DeSitter-DeSitter". First type of trajectories correspond to the trajectories populating region $I V$ in massless case, but their behaviour can be more involved due to passage through 
points of minimal contraction and maximal expansion. Classification of these trajectories in terms of configuration space $(a, \varphi)$ will be given in third section of this paper. The second type of trajectories corresponds to those in regions $I I$ and $I I I$ in the massless case while "DeSitter-DeSitter" trajectories correspond to those from the region $I$. These two types of trajectories will be studied in section 4 .

\section{Travelling between singularities}

It was shown in the preceding paper ${ }^{1}$ that the points of maximal expansion and those of minimal contraction can exist only in Euclidean region. In the case of the presence of cosmological constant $\Lambda$ the form of this region is given by equation

$$
\varphi^{2}<\frac{3 m_{P}^{2}}{4 a^{2}}-\frac{\Lambda m_{P}^{2}}{4 m^{2}}
$$

and this region is closed on the right at

$$
a=\sqrt{\frac{3}{\Lambda}}
$$

The area where located the possible points of maximal expansion is separated from that with points of minimal contraction by the curve

$$
\varphi^{2}=\frac{m_{P}^{2}}{2 \pi m^{2} a^{2}}-\frac{\Lambda m_{P}^{2}}{4 \pi m^{2}}
$$

which crosses the horizontal axis at

$$
a=\sqrt{\frac{2}{\Lambda}}
$$

Thus, geometry of this curve surrounding the region of possible points of maximal contraction differs from that consisting of two branches in the absence of $\Lambda$ (cf. Ref. 1). The configuration of Euclidean region and the separating curve (3.2) is shown in Fig. 2. 
In the preceding paper ${ }^{1}$ it was shown that the region of possible points of maximal expansion has quite a regular structure. In the left, closest to axis $a=0$ part of this region there are points of maximal expansion after which trajectory goes to singularity. Then one can see the region where after the going through the point of maximal expansion a trajectory undergoes the "bounce" i.e. goes through the point of minimal contraction. Then we have the region where after going through the point of maximal expansion trajectory has a " $\varphi$ - turn" i.e. has the extremum in the value of the scalar field $\varphi$ and then fells into singularity. Then one has the region corresponding to the trajectories having bounce after one oscillation in $\varphi$ and so on. Then studying the "substructure" of regions corresponding to trajectories possessing bounces, one can see that this substructure repeats the structure itself. In turn the "subsubstrucure" of regions having two bounces repeats the general structure and so on and so forth. Continuing this process ad infinitum we can get the set of infinitely bouncing trajectories escaping the singularity. It is plausible to believe that this set has a fractal nature ${ }^{11}$ due to recurrent type of the procedure of its construction ${ }^{5,1,9,12}$.

The remarkable feature of the model with the cosmological constant $\Lambda$ consists in the fact that this fractal structure of the set of infinitely bouncing trajectories survives provided the value of $\Lambda$ is small enough in comparison with the mass of scalar field.

An important distinction between the case of small $\Lambda \neq 0$ and $\Lambda=0$ consists in the fact that an area of all the bouncing intervals (i.e. such locations of points of maximal expansion after which the trajectories have bounce) is restricted from the right in configuration space by the new unstable periodical trajectory which has no analogue in the case of $\Lambda=0$. Trajectories situated on the left side of this periodical 
trajectory go to the regions of possible bounces while those situated on the right go to DeSitter point.Another distinction is that substructure of all intervals contains subintervals, corresponding to trajectories, going to DeSitter after bounce.

At some critical value of

$$
\Lambda \sim 0.5 m^{2}
$$

given by the numerical computation the complicated structure of subregions of the part of Euclidean region containing the points of maximal expansion vanishes. Instead one has only two regions (see Fig. 3): the left one containing the points of maximal expansion after which trajectories fell to singularity and the right one where after going through point of maximal expansion and then via point of minimal contraction trajectories go to DeSitter regime. Thus for the case of large values of $\Lambda$ the behaviour of trajectories qualitatively reminds that for the case of massless scalar field described in the preceding section.

It is interesting to notice that the transition from the chaotic (fractal) structure of trajectories when we have infinite number of subregions to the regular structure (Fig. 3) with two subregions has jump-like character. There is not transitional situation when one can see finite number of different subregions.

\section{Configuration of trajectories in the phase space}

In this section we consider the relation between the trajectories travelling between two DeSitter regimes and those which begin at DeSitter point and end in the singularity or viceversa. The boundary between these two classes of trajectories is rather complicated and fractal-like two dimensional surface in three-dimensional phase space. To describe it we shall use smooth two-dimensional projections ignor- 
ing its fractal nature.

It is convenient to introduce the following coordinates in the phase space:

$$
x=m_{P} \varphi, \quad y=\frac{m_{P} \varphi}{m}, \quad z=m \frac{\dot{a}}{a} .
$$

At $\Lambda \neq 0$ we have two De-Sitter points at $x=0$

$y=0$

$z= \pm m \sqrt{\Lambda / 3}$ Some part of trajectories beginning in the neighborhood of DeSitter points go to another DeSitter point while the rest of trajectories fell to singularity or in terms of phase space to an infinite sphere (see Refs. 2,3). To distinguish between these two classes of trajectories we make numerical integration of equations of motion starting from lower hemisphere around De-Sitter point. For concreteness, the radius of this hemisphere is chosen as $r=z / 3$. The behaviour of trajectories is shown in Fig. 4. It is convenient to parametrize initial conditions for trajectories on hemisphere by two angles $\phi$ and $\theta$ which are defined as

$$
\tan (\phi)=y / x ; \quad \sin (\theta)=(\sqrt{\Lambda / 3}+z) / r .
$$

While $t \rightarrow \infty$ a trajectory may go to singularity or to DeSitter asymptotic point. In Fig. 4 dashed area corresponds to trajectories going to singularity. It is necessary to emphasizes that this picture is a rough one - it exists a fine structure of trajectories escaping sungularity inside the dashed area due to bounces. In Fig. 4b such a structure is remarkable. For every value of relation $\Lambda / m^{2}$ there are two distinguished values of an angle $\theta: \theta_{\min }$ such that at $\theta<\theta_{\min }$ all the trajectories go to singularity independently of value of angle $\phi$ and $\theta_{\max }$ such that all the trajectories go to the second DeSitter point while $\theta>\theta_{\max }$.

It is interesting to trace out the dependence of these angles on the relation $\Lambda / m^{2}$. At small values of $\Lambda / m^{2}$ these angles almost coincide $\theta_{\min } \approx \theta_{\max }$. This 
fact can be explained by the fact that the distance between DeSitter points in the phase space is large in comparison with mass parameter and the trajectories manage to have so many rotations around axis $z$ that the dependence of their final destiny on the initial angle $\phi$ disappear. It would be reasonable to expect that in the limit $\Lambda / m^{2} \rightarrow \infty$ these two limiting angles should also coincide giving the angle separating different type of trajectories in massless case (cf. sec. 2), however it is not the case because in our parametrization small values of mass $m$ are compensated by possible large values of the field $\varphi$ and one does not have smooth transition to the massless model. Therefore, it is interesting to carry out numerical investigation of massless model considering since the beginning two-dimensional phase space. In this case one has only one parameter for the trajectories going from lower DeSitter point. It is "latitude" angle $\theta$. The value of $\theta$ separating trajectories going to singularity in the limit $r \rightarrow 0$ appears to be equal

$$
\theta_{\text {numerical }} \approx 1.2 \text {. }
$$

One can easily estimate theoretical value of this angle connecting DeSitter point with one of two saddle points of two-dimensional phase diagram (see Fig. 1). Using Eqs. (2.5) together with normalization given by Eqs. (4.1) one can easily get

$$
\theta_{\text {theor }}=\arctan \sqrt{\frac{8 \pi}{3}} \approx 1.3 \text {. }
$$

Thus we have seen that the results of theoretical consideration and numerical simulation are in a good agreement.

\section{Acknowledgement}

This work was supported by Russian Foundation for Basic Research via grants No 96-02-16220 and No 96-02-17591. 
1. A.Yu. Kamenshchik, I.M. Khalatnikov and A.V. Toporensky, Int. J. Mod. Phys. D, to appear, // gr-qc/9801064.

2. V.A. Belinsky, L.P. Grishchuk, Ya.B. Zel'dovich and I.M. Khalatnikov, J. Exp. Theor. Phys. 89, 346 (1985).

3. V.A. Belinsky and I.M. Khalatnikov, J. Exp. Theor. Phys. 93, 784 (1987); V.A. Belinsky, H. Ishihara, I.M. Khalatnikov and H. Sato, Progr. Theor. Phys. 79, $676(1988)$.

4. G.A. Burnett, Phys. Rev. D51, 1621 (1995).

5. D.N. Page, Class. Quantum Grav. 1, 417 (1984).

6. L. Parker and S.A. Fulling, Phys. Rev. D7, 2357 (1973); A.A. Starobinsky, Pisma A.J. 4155 (1978).

7. A. Melchiori, M. Sazhin, V. Shulga and N. Vittorio, in preparation; V.N. Lukash and E.V. Micheeva, Gravitation and Cosmology 2247 (1996).

8. V.A. Belinsky and I.M. Khalatnikov, J. Exp. Theor. Phys. 63, 1121 (1972).

9. I.M. Khalatnikov and A.Yu. Kamenshchik, Phys. Rep., to appear.

10 E.S. Nikomarov and I.M. Khalatnikov, ZhETF 75, 1176 (1978); I.M. Khalatnikov and V.A. Belinsky, Qualitative Cosmology in Problems of modern experimental and theoretical physics, dedicated to 80th anniversary of Yu.B. Khariton, ed. A.P. Alexandrov, Leningrad, 1984, p. 299 (in Russian).

11. B. Mandelbrot, The fractal geometry of nature, (San Francisco, Freeman, 1982).

12. N.J.Cornish, E.P.S.Shellard "Chaos in quantum cosmology" // gr-qc/9708046 


\section{Captions to Figures}

Fig. 1 In Fig. 1 the phase diagram for the model with massless scalar field is presented. Two hyperbolah going thgrough points $A$ and $C$ separate the part of phase space accessible for the trajectories of closed and open Friedmann - Robertson - Walker model. Points $A$ and $C$ are attracting and repelling nodes respectively, while points $B$ and $D$ are saddle ones. The region $I$ bounded by trajectories connecting the points $A$ and $B, B$ and $C, C$ and $D, D$ and $A$ is covered by trajectories which begins at point $C$, i.e since contraction according to DeSitter exponential law, then have a bounce and end in the point $A$ corresponding to DeSitter expansion. The region $I I$ contains trajectories expanding from singularity until DeSitter regime while the region $I I I$ contains trajectories contracting from DeSitter point $C$ to singularity. Finally, the region $I V$ contains trajectories which begin expansion from singularity, then go through the points of maximal expansion and then return to singularity again. The regions $I I$ and $I I I$ are separated from the region $I V$ by trajectories connecting saddle points $B$ and $D$ with singularity.

Fig. 2. Solid line denotes the boundary between Lorentz and Euclidean regions, dashed line corresponds to the curve separating the points of minimal contraction and maximal expansion.

Fig. 3. Separating curve (solid) and boundary of bouncing interval (dashed) in the case of large $\Lambda / m^{2}$. The bouncing interval is located between these two curves. Fig. 4 In Fig. 4 dashed area corresponds to the trajectories going out DeSitter contracting point and falling to singularity. The angles $\theta$ and $\varphi$ parametrize the direction of the trajectory in the initial DeSitter point. White area corresponds to trajectories going between two DeSitter points. In Fig. $4 \mathrm{a} \Lambda=0.05 \mathrm{~m}^{2}$, in Fig. 4b $\Lambda=0.5 m^{2}$, in Fig. $4 \mathrm{c} \Lambda=50 m^{2}$. 
This figure "lambda1.gif" is available in "gif" format from: http://arxiv.org/ps/gr-qc/9801082v1 


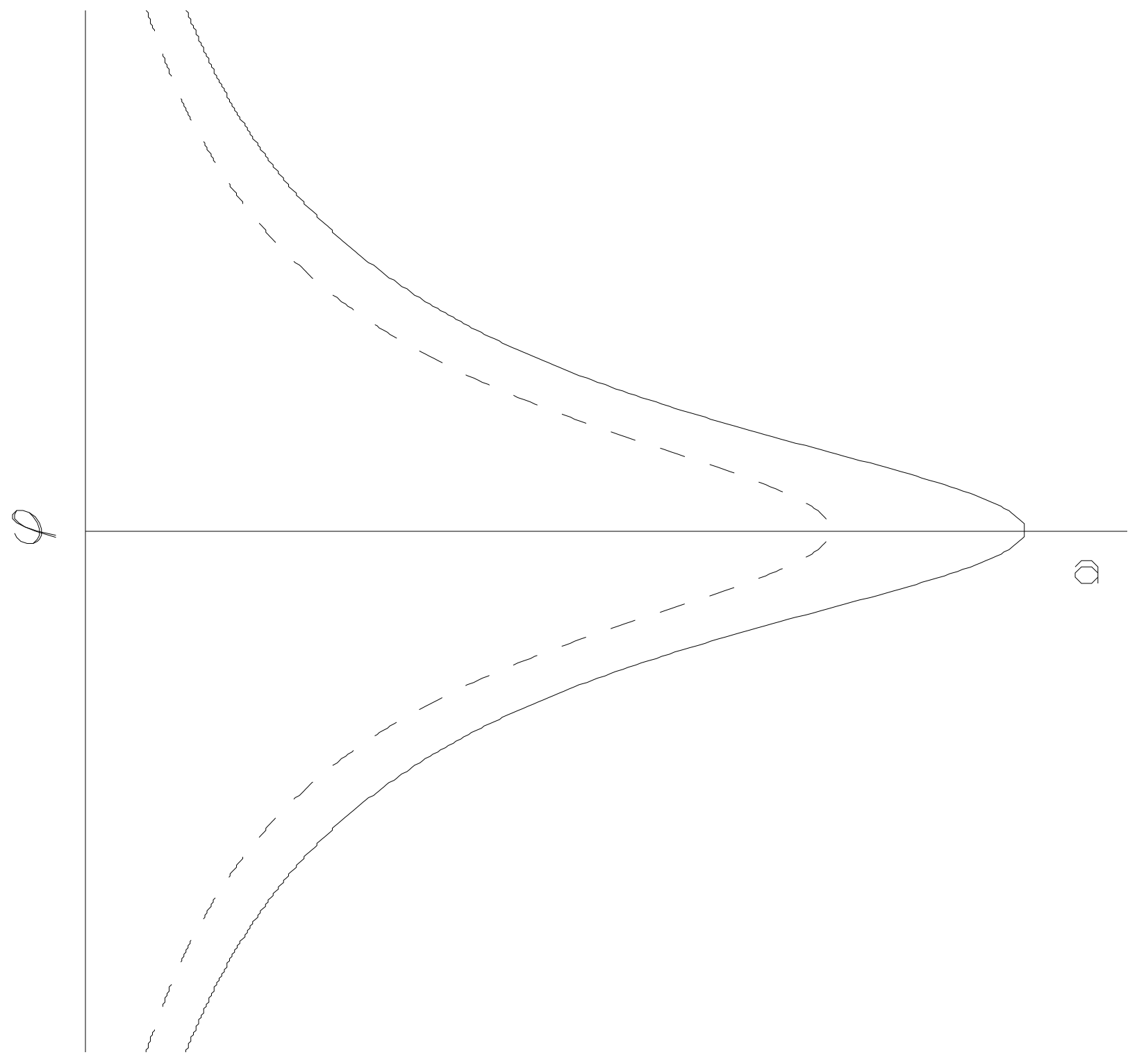




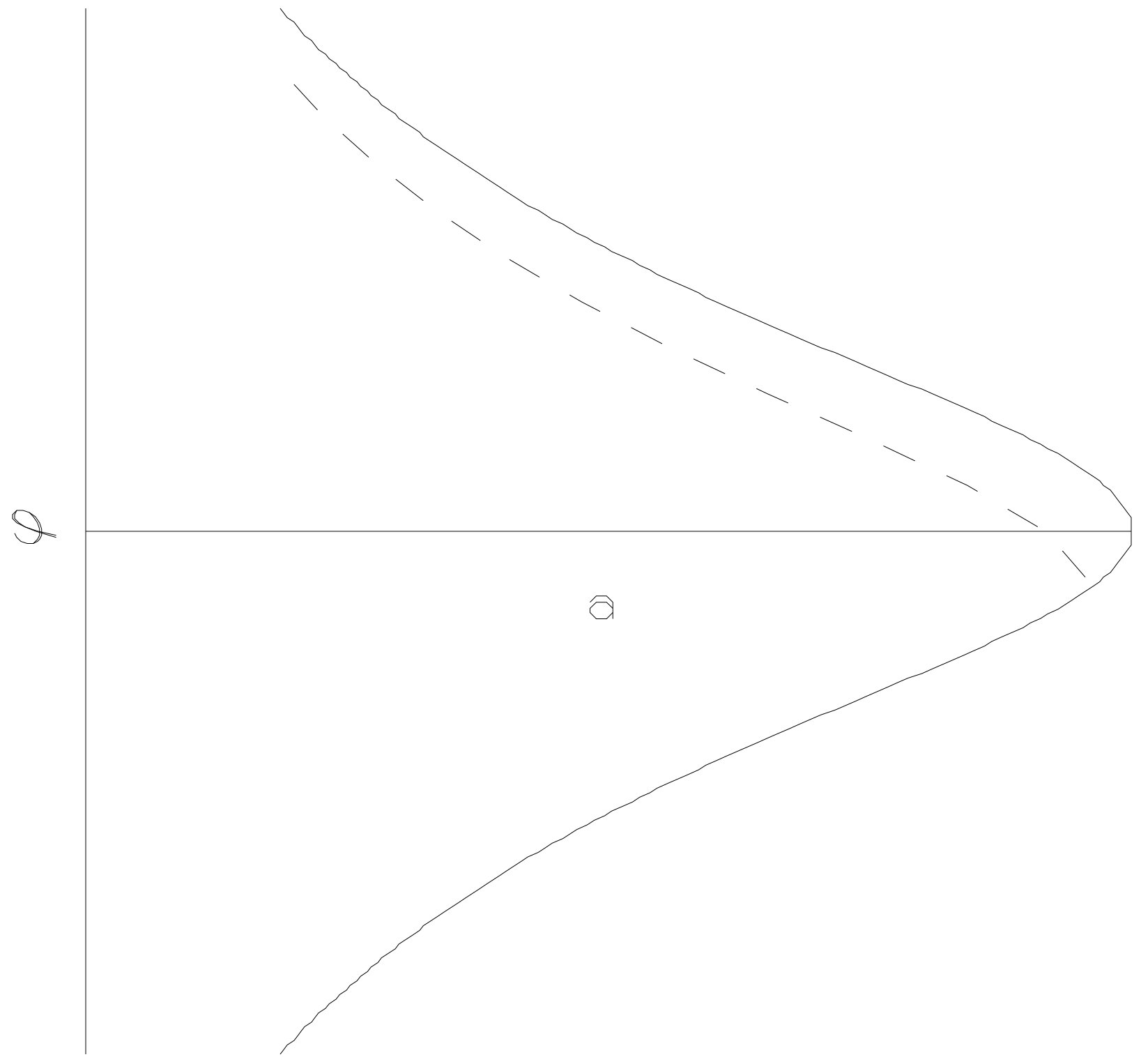


This figure "lambda4.gif" is available in "gif" format from: http://arxiv.org/ps/gr-qc/9801082v1 